\title{
一般薄膜方程的不变集和不变解
}

\section{屈长征 ${ }^{1,2} \quad$ 朱春蓉 $^{3}$}

(1. 西北大学非线性科学研究中心, 西安 710069; 2. 西北大学数学系, 西安 710069;

3. 安徽师范大学数学系, 芜湖 241000)

摘要主要讨论了 $1+2$ 维一般薄膜方程的不变集和不变解. 证明了对于这类方程, 有一 族解在集合

$$
E_{0}=\left\{u: u_{x}=v_{x} F(u), u_{y}=v_{y} F(u)\right\}
$$

中不变, 其中 $v$ 为关于 $x$ 和 $y$ 的光滑函数, $F$ 为 $u$ 的光滑函数. 文中的结果推广了 Galaktionov 中关于 $1+1$ 维非线性发展方程的结论.

关键词 薄膜方程 不变集 不变解 旋转群 伸缩群

$\operatorname{MSC}(2000)$ 主题分类 $35 \mathrm{~K} 55,35 \mathrm{~K} 40,82 \mathrm{C} 26$

\section{1 引言}

群不变解在研究偏微分方程解的长时间行为中起着重要作用, 其中自相似解对应于方程 的伸缩不变性, 即自相似解可以用伸缩不变性得到. 但是很多非线性偏微分方程不具有伸缩 不变性. 对不具有伸缩不变性的非线性偏微分方程, 为了得到更多的解, 有必要对普通的伸缩 群进行推广. 在文献 [1] 中, Galaktionov 对非线性演化方程

$$
u_{t}=A(u)=A\left(x, u_{x}, u_{x x}, \ldots, u^{(k)}\right),
$$

引进了不变集 $S_{0}=\left\{u: u_{x}=F(u) / x\right\}$, 其中 $F$ 是 $u$ 的适当光滑函数, 它由方程 (1) 在集合 $S_{0}$ 中的不变性确定. 这种方法可用于讨论二阶和四阶的非线性演化方程 (参见文献 [1]), 并 且此方法对研究非线性 $\mathrm{KdV}$ 型发展方程也比较有效 (参见文献 [2]). 在文献 [3] 中, 屈长征和 Estevez 将伸缩群进一步推广为一般形式, 其中对应的不变集为

$$
S_{1}=\left\{u: u_{x}=\frac{1}{x} F(u)+\varepsilon F(u)\left[\exp (n-1) \int^{u} \frac{1}{F(z)} d z\right]\right\} .
$$

在文献 [4] 中, 屈长征把这种方法用于讨论由液态薄膜产生的 $1+1$ 维的薄膜方程. 关于 $1+1$ 维的薄膜方程已有几种推广, 在文献 [5] 中, King 提出两类一般的薄膜方程, 这两类方程 与流体薄膜的毛细管作用流有关. 他通过拟设方法得到一些有趣的精确解. 从文献 [6-27] 也 可以看到其他一些与对称相关的方法成功地用于研究非线性偏微分方程 (1) 的解和对称性质. 本文讨论 $1+2$ 维的一般薄膜方程, 其相应的不变集是 $E_{0}=\left\{u: u_{x}=v_{x} F(u), u_{y}=v_{y} F(u)\right\}$. 我们的方法推广了 Galaktionov ${ }^{[1,2]}$ 的方法. 事实上,该不变集是文献 [1] 中 $S_{0}$ 的推广. 
考虑一般薄膜方程

$$
u_{t}=-\operatorname{div}\left(A(u) \triangle \nabla u+B(u) \nabla u \triangle u+C(u)|\nabla u|^{2} \nabla u\right)+Q(u),
$$

其中 $\nabla u=\left(u_{x}, u_{y}\right), \Delta u=u_{x x}+u_{y y}, A, B, C$ 和 $Q$ 为 $u$ 的光滑函数.

引进不变集

$$
E_{0}=\left\{u: u_{x}=v_{x} F(u), u_{y}=v_{y} F(u)\right\},
$$

其中 $v$ 是关于变量 $x$ 和 $y$ 的光滑函数, $F$ 是由不变条件

$$
u(x, y, 0) \in E_{0} \Longrightarrow u(x, y, t) \in E_{0}(t \in(0,1])
$$

确定的. 当 $u \in E_{0}$ 时, 我们得到方程形如

$$
\int^{u} \frac{d z}{F(z)}=v(x, y)+h(t)
$$

的解. 此解为一类函数变量分离解, 它可以看作对 $1+1$ 维情形中函数变量分离解

$$
\int^{u} \frac{1}{F(z)} d z=f(x)+g(t)
$$

的推广. 当 $u \in E_{0}$ 时, 有以下的公式:

$$
\begin{aligned}
u_{x}= & v_{x} F, u_{y}=v_{y} F, \quad u_{t}=h^{\prime}(t) F, \\
u_{x x}= & v_{x x} F+v_{x}^{2} F F^{\prime}, u_{y y}=v_{y y} F+v_{y}^{2} F F^{\prime}, u_{x y}=v_{x y} F+v_{x} v_{y} F F^{\prime}, \\
u_{x x x}= & v_{x x x} F+3 v_{x} v_{x x} F F^{\prime}+v_{x}^{3} F\left(F F^{\prime}\right)^{\prime}, \\
u_{y y y}= & v_{y y y} F+3 v_{y} v_{y y} F F^{\prime}+v_{y}^{3} F\left(F F^{\prime}\right)^{\prime}, \\
u_{x y y}= & v_{x y y} F+\left(2 v_{x y} v_{y}+v_{x} v_{y y}\right) F F^{\prime}+v_{x} v_{y}^{2} F\left(F F^{\prime}\right)^{\prime}, \\
u_{x x y}= & v_{x x y} F+\left(2 v_{x y} v_{x}+v_{y} v_{x x}\right) F F^{\prime}+v_{y} v_{x}^{2} F\left(F F^{\prime}\right)^{\prime}, \\
u_{x x x x}= & v_{x x x x} F+\left(4 v_{x x x} v_{x}+3 v_{x x}^{2}\right) F F^{\prime}+6 v_{x}^{2} v_{x x} F\left(F F^{\prime}\right)^{\prime}+v_{x}^{4} F\left(F\left(F F^{\prime}\right)^{\prime}\right)^{\prime}, \\
u_{y y y y}= & v_{y y y y} F+\left(4 v_{y y y} v_{y}+3 v_{y y}^{2}\right) F F^{\prime}+6 v_{y}^{2} v_{y y} F\left(F F^{\prime}\right)^{\prime}+v_{y}^{4} F\left(F\left(F F^{\prime}\right)^{\prime}\right)^{\prime}, \\
u_{x x y y}= & v_{x x y y} F+\left(2 v_{x y}^{2}+2 v_{y} v_{x x y}+2 v_{x} v_{x y y}+v_{x x} v_{y y}\right) F F^{\prime} \\
& +\left(v_{y}^{2} v_{x x}+v_{x}^{2} v_{y y}+4 v_{x} v_{y} v_{x y}\right) F\left(F F^{\prime}\right)^{\prime}+v_{x}^{2} v_{y}^{2} F\left(F\left(F F^{\prime}\right)^{\prime}\right)^{\prime} .
\end{aligned}
$$

将它们代入到 (2) 式中, 并利用 (4) 式得到

$$
\begin{aligned}
h^{\prime}(t)=- & \left\{A\left[\left(v_{x x}+v_{y y}\right)_{x x}+\left(v_{x x}+v_{y y}\right)_{y y}\right]\right. \\
& +\left(4 A F^{\prime}+A^{\prime} F+B F\right)\left[v_{x}\left(v_{x x}+v_{y y}\right)_{x}+v_{y}\left(v_{x x}+v_{y y}\right)_{y}\right] \\
& +\left(3 A F^{\prime}+B F\right)\left(v_{x x}+v_{y y}\right)^{2}-4 A F^{\prime}\left(v_{x x} v_{y y}-v_{x y}^{2}\right) \\
& +\left[\left(A F F^{\prime}+B F^{2}\right)^{\prime}+A\left(F F^{\prime}\right)^{\prime}+B F F^{\prime}+C F^{2}\right]\left(v_{x}^{2}+v_{y}^{2}\right)\left(v_{x x}+v_{y y}\right) \\
& +2\left[\left(A F F^{\prime}\right)^{\prime}+A\left(F F^{\prime}\right)^{\prime}+B F F^{\prime}+C F^{2}\right]\left(v_{x}^{2} v_{x x}+v_{y}^{2} v_{y y}+2 v_{x} v_{y} v_{x y}\right) \\
& \left.+\left[A F\left(F F^{\prime}\right)^{\prime}+B F^{2} F^{\prime}+C F^{3}\right]\left(v_{x}^{2}+v_{y}^{2}\right)^{2}\right\}+\frac{Q}{F} .
\end{aligned}
$$

从上面的关系式, 可以看出与方程 (1) 和 $E_{0}$ 有关的函数很难确定. 在第 2 节中, 我们将讨论 一些特殊情形. 


\section{2 主要结论}

在这一节中, 我们通过选取特殊的 $v(x, y)$ 来确定不变集和构造方程 $(1)$ 相应的解. 从上 节中, 可以看出我们不能确定所有的 $v(x, y)$ 使得方程 (1) 在 $E_{0}$ 中不变, 所以仅考虑下面一 些特殊情形:

情形 $1 \quad v_{x x}+v_{y y}=0$.

如果函数 $v(x, y)$ 满足方程

$$
v_{x x}+v_{y y}=0
$$

则 (5) 式可以简化为

$$
\begin{aligned}
h^{\prime}(t)=- & \left\{-4 A F^{\prime}\left(v_{x x} v_{y y}-v_{x y}^{2}\right)\right. \\
& +2\left[\left(A F F^{\prime}\right)^{\prime}+A\left(F F^{\prime}\right)^{\prime}+B F F^{\prime}+C F^{2}\right]\left(v_{x}^{2} v_{x x}+v_{y}^{2} v_{y y}+2 v_{x} v_{y} v_{x y}\right) \\
& \left.+\left[A F\left(F F^{\prime}\right)^{\prime}+B F^{2} F^{\prime}+C F^{3}\right]^{\prime}\left(v_{x}^{2}+v_{y}^{2}\right)^{2}\right\}+\frac{Q}{F} .
\end{aligned}
$$

利用方程 (6) 的解, 我们可以得到下面的一些结果:

情形 $1.1 \quad v(x, y)=a x+b y(a \neq 0, b \neq 0)$.

将 $v(x, y)=a x+b y$ 代入 (7) 式, 有

$$
h^{\prime}(t)=-\left(a^{2}+b^{2}\right)^{2}\left[A F\left(F F^{\prime}\right)^{\prime}+B F^{2} F^{\prime}+C F^{3}\right]^{\prime}+\frac{Q}{F} .
$$

由于 (8) 式左端与 $x$ 和 $y$ 无关, 因此 $A, B, C, Q, F$ 满足

$$
-\left(a^{2}+b^{2}\right)^{2}\left[A F\left(F F^{\prime}\right)^{\prime}+B F^{2} F^{\prime}+C F^{3}\right]^{\prime}+\frac{Q}{F}=c_{1},
$$

在这里以及下文中 $c_{i}$ 表示任意常数. 方程 $(9)$ 则给出方程 $(1)$ 中系数的约束条件. 在这种情 形下, 方程 (1) 有精确解

$$
\int^{u} \frac{d z}{f(z)}=a x+b y+c_{1} t+c_{2}
$$

此解为行波解.

情形 $1.2 \quad v_{x x}=v_{y y}=0$.

由 $v_{x x}=v_{y y}=0$, 我们得到解 $v(x, y)=c x y+d$. 不失一般性, 取 $c=1, d=0$. 在这种情 形下, $E_{0}$ 为集合 $E_{1}=\left\{u: u_{x}=y F(u), u_{y}=x F(u)\right\}$. 将 $v=x y$ 代入 (7) 式有

$$
h^{\prime}(t)=D(u)\left(x^{2}+y^{2}\right)^{2}+E(u) x y+G(u),
$$

其中

$$
\begin{aligned}
& D(u)=-\left[A F\left(F F^{\prime}\right)^{\prime}+B F^{2} F^{\prime}+C F^{3}\right]^{\prime}, \\
& E(u)=-4\left[\left(A F F^{\prime}\right)^{\prime}+A\left(F F^{\prime}\right)^{\prime}+B F F^{\prime}+C F^{2}\right], \\
& G(u)=-4 A F^{\prime}+\frac{Q}{F} .
\end{aligned}
$$

注意到 (10) 式左端不依赖于 $x$ 和 $y$, 故有

$$
\begin{aligned}
& D^{\prime} F y\left(x^{2}+y^{2}\right)^{2}+\left(4 D+E^{\prime} F\right) x y^{2}+4 D x^{3}+\left(E+G^{\prime} F\right) y=0, \\
& D^{\prime} F x\left(x^{2}+y^{2}\right)^{2}+\left(4 D+E^{\prime} F\right) y x^{2}+4 D y^{3}+\left(E+G^{\prime} F\right) x=0 .
\end{aligned}
$$


由这两个方程得到

$$
D=0, \quad E=c_{1}, \quad G=-c_{1} \int^{u} \frac{d z}{F(z)}+c_{2},
$$

其中 $h(t)$ 满足 $h^{\prime}(t)=-c_{1} h(t)+c_{2}$. 将 (12) 式代入 (11) 式中有

$$
\begin{aligned}
& A F\left(F F^{\prime}\right)^{\prime}+B F^{2} F^{\prime}+C F^{3}=c_{3}, \\
& \left(A F F^{\prime}\right)^{\prime}+A\left(F F^{\prime}\right)^{\prime}+B F F^{\prime}+C F^{2}=-\frac{c_{1}}{4}, \\
& \frac{Q}{F}-4 A F^{\prime}=-c_{1} \int^{u} \frac{d z}{F(z)}+c_{2} .
\end{aligned}
$$

这个方程组等价于关系式

$$
\begin{aligned}
& A\left(F^{2}\right)^{\prime \prime}+B\left(F^{2}\right)^{\prime}+2 C F^{2}=\frac{2 c_{3}}{F}, \\
& A\left(F^{2}\right)^{\prime}=-2 c_{3} \int^{u} \frac{d z}{F(z)}-\frac{c_{1}}{2} u+c_{4}, \\
& Q=-\left(c_{1} F+4 c_{3}\right) \int^{u} \frac{d z}{F(z)}+c_{2} F-c_{1} u+2 c_{4} .
\end{aligned}
$$

现在考虑 $A=u^{m},(m \neq 0)$ 时的一些情形:

当 $m<2$ 时, 令 $c_{1}=-2, c_{2}=c_{3}=c_{4}=0, C=0$, 有 $F=u^{1-m / 2} / \sqrt{2-m}$, $B=(m-1) u^{m-1}$. 这证明了方程

有精确解

$$
u_{t}=-\operatorname{div}\left(u^{m} \triangle \nabla u+(m-1) u^{m-1} \nabla u \triangle u\right)+\frac{4}{m}+2 u,(m<2)
$$

$$
u=\left[\frac{m}{2 \sqrt{2-m}}\left(x y+e^{2 t}\right)\right]^{\frac{2}{m}} .
$$

当 $0<m<2$ 时, 解 $u$ 在集合 $S=\left\{(x, y), x y=-e^{2 t}\right\}$ 上熄灭.

当 $m<0$ 时,解 $u$ 在集合 $S=\left\{(x, y), x y=-e^{2 t}\right\}$ 上爆破.

当 $m=-2$ 时, 方程 $u_{t}=-\operatorname{div}\left(u^{-2} \triangle \nabla u-3 u^{-3} \nabla u \triangle u\right)$ 有精确解

$$
u=-\frac{2}{x y+e^{2 t}} \text {. }
$$

当 $m=1$ 时, 方程 $u_{t}=-\operatorname{div}(u \triangle \nabla u)+6 u$ 有精确解

$$
u=\left(\frac{x y+e^{2 t}}{2}\right)^{2} \text {. }
$$

当 $m=2$ 时, 设 $c_{1}=-2, c_{2}=c_{3}=c_{4}=0, C=0$, 则 $F=\sqrt{\ln u}$. 此时可以证明方程

有精确解

$$
u_{t}=-\operatorname{div}\left(u^{m} \triangle \nabla u+u^{m-1} \nabla u \triangle u\right)+2 \sqrt{\ln u}\left(\int^{u} \frac{d z}{\sqrt{\ln z}}+1\right)
$$

$$
\int^{u} \frac{d z}{\sqrt{\ln z}}=x y+e^{2 t}
$$

当 $m>2$ 时, 设 $c_{1}=2, c_{2}=c_{3}=c_{4}=0, C=0$, 有 $F=u^{1-\frac{m}{2}} / \sqrt{m-2}$. 易证方程

有精确解

$$
u_{t}=-\operatorname{div}\left(u^{m} \triangle \nabla u+(m-1) u^{m-1} \nabla u \triangle u\right)-\left(\frac{4}{m}+2\right) u,(m>2)
$$

$$
u=\left[\frac{m}{2 \sqrt{m-2}}\left(x y+e^{-2 t}\right)\right]^{\frac{2}{m}}
$$


记 $H=H(u)=\int^{u} \frac{d z}{F(z)}$, 即 $F(u)=\frac{1}{H^{\prime}(u)}$, 因此当 $Q=0$ 时, $H(u)$ 满足

$$
-c_{1} \frac{H}{H^{\prime}}+c_{2} \frac{1}{H^{\prime}}-4 c_{3} H-c_{1} u+2 c_{4}=0,
$$

解得

$$
H(u)= \begin{cases}\frac{c_{2} u-c_{0}}{c_{1} u-2 c_{4}}, & c_{3}=0, \\ \frac{-c_{1} u+2 c_{4}+\sqrt{\left(c_{1} u-2 c_{4}\right)^{2}+8 c_{3}\left(c_{2} u-c_{0}\right)}}{4 c_{3}}, & c_{3} \neq 0,\end{cases}
$$

从而当方程 $(1)$ 中系数满足

$$
\begin{aligned}
& A(u)=\frac{1}{2} c_{3} u^{-4}-\frac{1}{2} c_{2} c_{3} u^{-3}-\frac{1}{8} u^{-2} \\
& B(u)=-c_{3} u^{-5}+\frac{3}{2} c_{3} c_{2} u^{-4}+\frac{3}{8} u^{-3} \\
& C(u)=Q(u)=0
\end{aligned}
$$

或者

$$
\begin{aligned}
A(u) & =\frac{1}{2} c_{3} u^{-4}-\frac{1}{2} c_{2} c_{3} u^{-3}-\frac{1}{8} u^{-2} \\
C(u) & =\frac{3}{4} u^{-4}+3 c_{2} c_{3} u^{-5}-2 c_{3} u^{-6} \\
B(u) & =Q(u)=0
\end{aligned}
$$

时, 方程 (1) 有解

$$
u=\frac{1+2\left[c_{3} x y+\left(c_{2}+a e^{-t}\right)\right]^{2}}{-x y-a e^{-t}},
$$

其中 $a$ 为任意常数.

当 $F(u)$ 满足方程组 (14) 中前两个方程以及 $(15)$ 式时, 方程

$$
u_{t}=-\operatorname{div}\left(A(u) \triangle \nabla u+B(u) \nabla u \triangle u+C(u)|\nabla u|^{2} \nabla u\right)
$$

有精确解

$$
H(u)=\int^{u} \frac{d z}{F(z)}=x y+h(t)
$$

这里 $h(t)$ 满足方程 $h^{\prime}(t)=-c_{1} h(t)+c_{2}$, 而我们已经证明方程 $u_{t}=-\operatorname{div}\left(u^{-2} \triangle \nabla u-3 u^{-3} \nabla u \triangle u\right)$ 有精确解

$$
u=-\frac{2}{x y+e^{2 t}} .
$$

情形 $1.3 \quad v(x, y)=\ln \sqrt{x^{2}+y^{2}}$.

注意到函数 $v(x, y)=\ln \sqrt{x^{2}+y^{2}}$ 为方程 $(6)$ 的基本解. 在这种情形下, $E_{0}$ 为集合 $E_{2}=$ $\left\{u: u_{x}=\left(x /\left(x^{2}+y^{2}\right)\right) F(u), u_{y}=\left(y /\left(x^{2}+y^{2}\right)\right) F(u)\right\}$. 将 $v(x, y)=\ln \sqrt{x^{2}+y^{2}}$ 代入 (7) 式, 有

$$
h^{\prime}(t)=-D(u) \frac{1}{\left(x^{2}+y^{2}\right)^{2}}+\frac{Q}{F},
$$

其中

$$
\begin{aligned}
D(u)= & 4 A F^{\prime}-2\left[\left(A F F^{\prime}\right)^{\prime}+A\left(F F^{\prime}\right)^{\prime}+B F F^{\prime}+C F^{2}\right] \\
& +\left[A F\left(F F^{\prime}\right)^{\prime}+B F^{2} F^{\prime}+C F^{3}\right]^{\prime} .
\end{aligned}
$$


注意到 (16) 式左端不依赖于 $x$ 和 $y$, 对 (16) 式两端分别关于 $x$ 和 $y$ 求偏导有

解得

$$
\begin{aligned}
& -D^{\prime} F \frac{x}{\left(x^{2}+y^{2}\right)^{3}}+D \frac{4 x}{\left(x^{2}+y^{2}\right)^{3}}+\left(\frac{Q}{F}\right)^{\prime} F \frac{x}{\left(x^{2}+y^{2}\right)}=0, \\
& -D^{\prime} F \frac{y}{\left(x^{2}+y^{2}\right)^{3}}+D \frac{4 y}{\left(x^{2}+y^{2}\right)^{3}}+\left(\frac{Q}{F}\right)^{\prime} F \frac{y}{\left(x^{2}+y^{2}\right)}=0,
\end{aligned}
$$

$$
D=c_{0} e^{4 \int^{u} \frac{d z}{F(z)}}, Q=c_{1} F,
$$

并且 $h(t)$ 满足 $h^{\prime}(t)=-c_{0} e^{4 h(t)}+c_{1}$. 将 $D(u)$ 改写成 $D(u)=2 E(u)-[F E(u)]^{\prime}$, 其中 $E(u)$ 为 $E(u)=2 A F^{\prime}-\left(A\left(F F^{\prime}\right)^{\prime}+B F F^{\prime}+C F^{2}\right)$. 再由 (18) 式得到

$$
E(u)=\frac{1}{F(u)} e^{2 \int^{u} \frac{d z}{F(z)}}\left(-c_{0} \int^{u} e^{2 \int^{v} \frac{d z}{F(z)}} d v+c_{2}\right) .
$$

设 $c_{0}=8, c_{1}=0, c_{2}=0, A=e^{4 \sqrt{u}}, C=0, F=2 \sqrt{u}$, 则 $B=2 e^{4 \sqrt{u}}$. 我们可以证明方程

$$
u_{t}=-\operatorname{div}\left(e^{4 \sqrt{u}} \triangle \nabla u+2 e^{4 \sqrt{u}} \nabla u \triangle u\right)
$$

有精确解

$$
u=\left[\frac{1}{2} \ln \left(x^{2}+y^{2}\right)-\frac{1}{4} \ln (32 t+a)\right]^{2},
$$

其中 $a$ 为任意常数.

同理, 可证方程

$$
u_{t}=-\operatorname{div}\left(u^{m} \triangle \nabla u+\frac{1}{2} u^{m-\frac{1}{2}} \triangle u \nabla u+\frac{1}{4} u^{m-\frac{3}{2}}|\nabla u|^{2} \nabla u\right)+2 u^{\frac{1}{2}},
$$

有精确解 $u=\left[\ln \sqrt{x^{2}+y^{2}}+t\right]^{2}$.

情形 $2 \quad v_{x y}=0$.

由 $v_{x y}=0$, 得 $v(x, y)=f(x)+g(y)$, 其中 $f(x)$ 和 $g(y)$ 分别为 $x$ 和 $y$ 的光滑函数. 在这 种情形下, $E_{0}$ 为集合

$$
E_{3}=\left\{u: u_{x}=f^{\prime}(x) F(u), u_{y}=g^{\prime}(y) F(u)\right\} .
$$

情形 2.1 旋转群.

取 $f(x)=x^{2} / 2, g(y)=y^{2} / 2$, 即 $f^{\prime}(x)=x, g^{\prime}(y)=y, E_{0}$ 转化为

$$
R_{1}=\left\{u: u_{x}=x F(u), u_{y}=y F(u)\right\} .
$$

此时的 $E_{0}$ 为 $1+1$ 维情形的推广, $R_{1}$ 为文献 [3] 中 $R_{0}$ 的推广. 将 $v=x^{2} / 2+y^{2} / 2$ 代入 (5) 式中, 有

$$
h^{\prime}(t)=D(u)\left(x^{2}+y^{2}\right)^{2}+E(u)\left(x^{2}+y^{2}\right)+G(u),
$$

其中

$$
\begin{aligned}
& D(u)=-\left[A F\left(F F^{\prime}\right)^{\prime}+B F^{2} F^{\prime}+C F^{3}\right]^{\prime}, \\
& E(u)=-2\left(2 A F F^{\prime}+B F^{2}\right)^{\prime}+4\left[A\left(F F^{\prime}\right)^{\prime}+B F F^{\prime}+C F^{2}\right], \\
& G(u)=-4\left(2 A F^{\prime}+B F\right)+\frac{Q}{F} .
\end{aligned}
$$

注意到 (20) 式左端不依赖于 $x$ 和 $y$, 由此得到下列方程:

$$
\begin{aligned}
& D^{\prime} F x\left(x^{2}+y^{2}\right)^{2}+\left(4 D+E^{\prime} F\right) x\left(x^{2}+y^{2}\right)+\left(2 E+G^{\prime} F\right) x=0, \\
& D^{\prime} F y\left(x^{2}+y^{2}\right)^{2}+\left(4 D+E^{\prime} F\right) y\left(x^{2}+y^{2}\right)+\left(2 E+G^{\prime} F\right) x=0 .
\end{aligned}
$$


这个方程组的解为

$$
D^{\prime}=0, \quad 4 D+E^{\prime} F=0, \quad 2 E+G^{\prime} F=0,
$$

即

$$
\begin{aligned}
D & =c_{1}, \\
E & =-4 c_{1} \int^{u} \frac{d z}{F(z)}+c_{2}, \\
G & =4 c_{1}\left(\int^{u} \frac{d z}{F(z)}\right)^{2}-2 c_{2} \int^{u} \frac{d z}{F(z)}+c_{3},
\end{aligned}
$$

这里 $h(t)$ 满足

$$
h^{\prime}(t)=4 c_{1} h^{2}(t)-2 c_{2} h(t)+c_{3} .
$$

将 (21) 式代入 (22) 式中, 有

$$
\begin{aligned}
& A\left(F^{2}\right)^{\prime \prime}+B\left(F^{2}\right)^{\prime}+C F^{2}=-2 c_{1} \frac{u}{F(u)}+2 c_{4} \frac{1}{F}, \\
& A\left(F^{2}\right)^{\prime}+B F^{2}=2\left(c_{1} u-c_{4}\right) \int^{u} \frac{d z}{F(z)}-\frac{1}{2} c_{2} u+c_{5}, \\
& Q=4 c_{1} F\left(\int^{u} \frac{d z}{F(z)}\right)^{2}-2 c_{2} F \int^{u} \frac{d z}{F(z)}+c_{2} F \\
& \quad+8\left(c_{1} u-c_{4}\right) \int^{u} \frac{d z}{F(z)}-2 c_{2} u+4 c_{5},
\end{aligned}
$$

记 $H=H(u)=\int^{u} \frac{d z}{F(z)}$, 即 $F(u)=\frac{1}{H^{\prime}(u)}$. 此时, 当 $Q=0$ 时, $H(u)$ 满足常微分方程

$$
4 c_{1} \frac{H^{2}}{H^{\prime}}-2 c_{2} \frac{H}{H^{\prime}}+c_{2} \frac{1}{H^{\prime}}+8\left(c_{1} u-c_{4}\right) H-2 c_{2} u+4 c_{5}=0,
$$

求解得

$$
H(u)=\frac{\left(c_{2} u-2 c_{5}\right) \pm \sqrt{\left(c_{2} u-2 c_{5}\right)^{2}-4\left(c_{1} u-c_{4}\right)\left(c_{2} u-c_{0}\right)}}{4\left(c_{1} u-c_{4}\right)} .
$$

在这种情形下, 我们可以证明方程

$$
u_{t}=-\operatorname{div}\left(A(u) \nabla \triangle u+B(u) \nabla u \triangle u+C(u)|\nabla u|^{2} \nabla u\right)
$$

有精确解

$$
H(u)=\int^{u} \frac{d z}{F(z)}=\frac{x^{2}+y^{2}}{2}+h(t)
$$

其中 $h(t)$ 满足

$$
h^{\prime}(t)=4 c_{1} h^{2}(t)-2 c_{2} h(t)+c_{3} .
$$

例如, 若令 $c_{1}=9, c_{0}=1, c_{2}=c_{3}=c_{4}=c_{5}=0$, 则 $H=(1 / 6) u^{-\frac{1}{2}}, F=-12 u^{3 / 2}$, 因此, 方程

$$
u_{t}=-\operatorname{div}\left(\frac{5}{28} u^{-\frac{3}{2}} \triangle \nabla u-\frac{1}{32} u^{-\frac{5}{2}} \nabla u \triangle u\right)
$$

有精确解

$$
u=\left[\frac{1}{3\left(x^{2}+y^{2}\right)-\frac{6}{36 t+c_{6}}}\right]^{2} .
$$

注意到在 $T_{0}$ 时刻, 此解在集合 $\left\{(x, y): x^{2}+y^{2}=2 /\left(36 T_{0}+C_{6}\right)\right\}$ 上爆破. 
设 $A=B, C=0, c_{1}=c_{2}=c_{4}=c_{5}=0$, 则对任意的 $A(u), F=e^{-u / 2}$ 满足方程组 $(23)$ 中的前两个方程, 这说明了方程

$$
u_{t}=-\operatorname{div}[A(u)(\triangle \nabla u+\triangle u \nabla u)]+c_{3} e^{-\frac{u}{2}}
$$

有精确解

$$
u=2 \ln \left(x^{2}+y^{2}+c_{3} t+c_{6}\right)-2 \ln 2 .
$$

设 $A=u^{m}, F=u^{1 / 2}, c_{1}=3, c_{2}=c_{3}=c_{4}=c_{5}=0$, , 则 $B=12 u^{1 / 2}-u^{m-1}, C=$ $\frac{1}{2} u^{m-2}-9 u^{-1 / 2}, Q=96 u^{3 / 2}$. 此时方程

$$
u_{t}=-\operatorname{div}\left[u^{m} \triangle \nabla u+\left(12 u^{1 / 2}-u^{m-1}\right) \triangle u \nabla u+\left(\frac{1}{2} u^{m-2}-9 u^{-\frac{1}{2}}\right)|\nabla u|^{2} \nabla u\right]+96 u^{\frac{3}{2}},
$$

有精确解

$$
u=\left(\frac{x^{2}+y^{2}}{4}-\frac{1}{24 t+c_{6}}\right)^{2},
$$

此解在集合 $\left\{(x, y): x^{2}+y^{2}=4 /\left(24 t+c_{6}\right)\right\}$ 上熄灭.

情形 2.2 伸缩群.

取 $f(x)=\ln |x|, g(y)=\ln |y|, E_{0}$ 为集合 $S_{2}=\left\{u: u_{x}=(1 / x) F(u), u_{y}=(1 / y) F(u)\right\}$, 此 集合是对 $S_{0}$ 的二维推广. 如果方程 (1) 在集合 $S_{2}$ 中不变, 则方程 (1) 有精确解

$$
\int^{u} \frac{d z}{F(z)}=\ln |x y|+h(t) .
$$

由 (25) 式, 我们直接得到下面的公式:

$$
\begin{aligned}
u_{x} & =\frac{1}{x} F, \quad u_{y}=\frac{1}{y} F, \quad u_{t}=h^{\prime}(t) F, \quad u_{x x}=\frac{1}{x^{2}} G_{2}(F), \\
u_{y y} & =\frac{1}{y^{2}} G_{2}(F), \quad u_{x y}=\frac{1}{x y}\left(G_{2}(F)+F\right), \quad u_{x x x}=\frac{1}{x^{3}} G_{3}(F), \\
u_{y y y} & =\frac{1}{y^{3}} G_{3}(F), \quad u_{x x y}=\frac{1}{x^{2} y}\left(G_{3}(F)+2 G_{2}(F)\right), \\
u_{x y y} & =\frac{1}{y^{2} x}\left(G_{3}(F)+2 G_{2}(F)\right), \quad u_{x x x x}=\frac{1}{x^{4}} G_{4}(F), \\
u_{y y y y} & =\frac{1}{y^{4}} G_{4}(F), \quad u_{x x y y}=\frac{1}{x^{2} y^{2}}\left(G_{4}(F)+4 G_{3}(F)+2 G_{2}(F)\right),
\end{aligned}
$$

其中

$$
\begin{aligned}
& G_{2}(F)=F\left(F^{\prime}-1\right), \quad G_{3}(F)=F G_{2}^{\prime}-2 G_{2}=F\left[\left(F F^{\prime}\right)^{\prime}-3 F^{\prime}+2\right], \\
& G_{4}(F)=F G_{3}^{\prime}-3 G_{3}=F\left[\left(F\left(F F^{\prime}\right)^{\prime}\right)^{\prime}-6\left(F F^{\prime}\right)^{\prime}+11 F^{\prime}-6\right] .
\end{aligned}
$$

将上述所有的式子代入 (2) 式, 得

$$
h^{\prime}(t)=D(u)\left(\frac{1}{x^{4}}+\frac{1}{y^{4}}\right)+E(u) \frac{1}{x^{2} y^{2}}+G(u),
$$

其中

$$
\begin{aligned}
D(u) & =-\left(A G_{3}+B F G_{2}+C F^{3}\right)^{\prime}+\frac{3}{F}\left(A G_{3}+B F G_{2}+C F^{3}\right), \\
E(u) & =-2\left(A F G_{2}^{\prime}+B F G_{2}+C F^{3}\right)^{\prime}+\frac{2}{F}\left(A F G_{2}^{\prime}+B F G_{2}+C F^{3}\right), \\
G(u) & =\frac{Q}{F} .
\end{aligned}
$$


由于 (26) 式左端不依赖于 $x$ 和 $y$, 因此对 (26) 式两端分别关于 $x$ 和 $y$ 求偏导, 得

$$
\begin{aligned}
& D^{\prime} F\left(\frac{1}{x^{4}}+\frac{1}{y^{4}}\right) \frac{1}{x}-4 D \frac{1}{x^{5}}+\left(E^{\prime} F-2 E\right) \frac{1}{x^{3} y^{2}}+G^{\prime} F \frac{1}{x}=0, \\
& D^{\prime} F\left(\frac{1}{x^{4}}+\frac{1}{y^{4}}\right) \frac{1}{y}-4 D \frac{1}{y^{5}}+\left(E^{\prime} F-2 E\right) \frac{1}{y^{3} x^{2}}+G^{\prime} F \frac{1}{y}=0,
\end{aligned}
$$

进一步化简得

$$
D=0, \quad E^{\prime} F-2 E=0, \quad G^{\prime} F=0
$$

即

$$
D=0, \quad E=c_{0} e^{2 \int^{u} \frac{d z}{F(z)}}, Q=c_{1} F,
$$

并且 $h(t)$ 满足 $h^{\prime}(t)=c_{0} e^{2 h(t)}+c_{1}$. 将 (27) 式代入 $(28)$ 式, 我们有

$$
\begin{aligned}
& A G_{3}+B F G_{2}+C F^{3}=c_{2} e^{3 \int^{u} \frac{d z}{F(z)}}, \\
& A F G_{2}^{\prime}+B F G_{2}+C F^{3}=e^{\int^{u} \frac{d z}{F(z)}}\left(-\frac{c_{0}}{2} \int^{u}\left(e^{\int^{v} \frac{d z}{F(z)}}\right) d v+c_{3}\right), \\
& Q=c_{1} F .
\end{aligned}
$$

设 $c_{0}=4, c_{1}=c_{2}=c_{3}=0, F=2 \sqrt{u}, C=0$, 则 $A=e^{2 \sqrt{u}}, B=[(2 \sqrt{u}-3) /(2 \sqrt{u}(\sqrt{u}-$ 1)) $] e^{2 \sqrt{u}}$. 易证方程

$$
u_{t}=-\operatorname{div}\left(e^{2 \sqrt{u}} \nabla \Delta u+\frac{2 \sqrt{u}-3}{2 \sqrt{u}(\sqrt{u}-1)} e^{2 \sqrt{u}} \nabla u \triangle u\right)
$$

有精确解 $u=\left(\ln |x y|+\frac{1}{2} \ln |a-8 t|\right)^{2}$, 其中 $a$ 为任意常数.

同理, 可证方程

$$
u_{t}=-\operatorname{div}\left[\frac{e^{3 \sqrt{u}}+e^{\sqrt{u}}}{\sqrt{u}-1} \Delta \nabla u+\frac{(-2 \sqrt{u}+3) e^{\sqrt{u}}+e^{3 \sqrt{u}}}{-2 \sqrt{u}(\sqrt{u}-1)^{2}} \nabla \Delta u\right]+2 \sqrt{u}
$$

有精确解

$$
u=(\ln |x y|+t)^{2} .
$$

情形 2.3 推广的伸缩旋转不变群.

为了给出伸缩和旋转群的统一推广, 引入不变集

$$
E_{4}=\left\{u: u_{x}=f^{\prime}(x) F(u), u_{y}=g^{\prime}(y) F(u)\right\},
$$

其中 $f^{\prime}(x), g^{\prime}(y)$ 分别满足

$$
\begin{aligned}
f^{\prime \prime}(x) & =a f^{\prime 2}(x)+b, \\
g^{\prime \prime}(y) & =a g^{\prime 2}(y)+b,
\end{aligned}
$$

其中 $a$ 和 $b$ 为不等于零的某些常数.

注意到当 $a=0$ 时, $E_{4}$ 变为 $R_{1}$, 当 $b=0$ 时, $E_{4}$ 为 $S_{1}$, 因此 $E_{4}$ 可以看作 $R_{1}$ 和 $S_{1}$ 的一 
般形式. 当 $u \in E_{4}$ 时, 我们有下列公式:

$$
\begin{aligned}
u_{x}= & f^{\prime} F, \quad u_{y}=g^{\prime} F, \\
u_{x x}= & \left(a f^{\prime 2}+b\right) F+f^{\prime 2} F F^{\prime}, u_{y y}=\left(a g^{2}+b\right) F+g^{\prime 2} F F^{\prime}, \\
u_{x x x}= & 2 a f^{\prime}\left(a f^{\prime 2}+b\right) F+3 f^{\prime}\left(a f^{\prime 2}+b\right) F F^{\prime}+f^{\prime 3} F\left(F F^{\prime}\right)^{\prime}, \\
u_{y y y}= & 2 a g^{\prime}\left(a g^{\prime 2}+b\right) F+3 g^{\prime}\left(a g^{\prime 2}+b\right) F F^{\prime}+g^{\prime 3} F\left(F F^{\prime}\right)^{\prime}, \\
u_{x x y}= & g^{\prime}\left(a f^{\prime 2}+b\right) F F^{\prime}+g^{\prime} f^{\prime 2} F\left(F F^{\prime}\right)^{\prime}, \\
u_{x y y}= & f^{\prime}\left(a g^{\prime 2}+b\right) F F^{\prime}+f^{\prime} g^{\prime 2} F\left(F F^{\prime}\right)^{\prime}, \\
u_{x x x x}= & \left(a f^{\prime 2}+b\right)\left(6 a^{2} f^{\prime 2}+2 a b\right) F+\left(a f^{\prime 2}+b\right)\left(11 a f^{\prime 2}+3 b\right) F F^{\prime} \\
& +\left(6 a f^{\prime 4}+6 b f^{\prime 2}\right) F\left(F F^{\prime}\right)^{\prime}+f^{\prime 4} F\left(F\left(F F^{\prime}\right)^{\prime}\right)^{\prime}, \\
u_{y y y y}= & \left(a g^{\prime 2}+b\right)\left(6 a^{2} g^{\prime 2}+2 a b\right) F+\left(a f^{\prime 2}+b\right)\left(11 a g^{\prime 2}+3 b\right) F F^{\prime} \\
& +\left(6 a g^{\prime 4}+6 b g^{\prime 2}\right) F\left(F F^{\prime}\right)^{\prime}+g^{\prime 4} F\left(F\left(F F^{\prime}\right)^{\prime}\right)^{\prime}, \\
u_{x x y y}= & \left(a f^{\prime 2}+b\right)\left(a g^{\prime 2}+b\right) F F^{\prime}+\left[2 a f^{\prime 2} g^{\prime 2}+b\left(f^{\prime 2}+g^{\prime 2}\right)\right] F\left(F F^{\prime}\right)^{\prime} \\
& +f^{\prime 2} g^{\prime 2} F\left(F\left(F F^{\prime}\right)^{\prime}\right)^{\prime} .
\end{aligned}
$$

将上述各式代入 (5) 式中得到

$$
h^{\prime}(t)=E(u)\left(f^{\prime 4}+g^{\prime 4}\right)+G(u)\left(f^{\prime 2}+g^{\prime 2}\right)+H(u) f^{\prime 2} g^{\prime 2}+I(u),
$$

其中

$$
\begin{aligned}
E(u) & =-\left\{6 a^{3} A+a^{2}\left(11 A F^{\prime}+2 A^{\prime} F+3 B F\right)+2 b\left[\left(3 A F F^{\prime}+B F^{2}\right)^{\prime}+3 D\right]+(D F)^{\prime}\right\}, \\
G(u) & =-\left\{8 a^{2} b A+2 a b\left(8 A F^{\prime}+A^{\prime} F+3 B F\right)+2 b\left[\left(2 A F F^{\prime}+B F^{2}\right)^{\prime}+2 D\right]\right\}, \\
H(u) & \left.=-\left\{2 a^{2}\left(A F^{\prime}+B F\right)+2 a\left[\left(F\left(A F^{\prime}+B F\right)\right)^{\prime}+D\right]+2(D F)^{\prime}\right]\right\}, \\
I(u) & =-4 a^{2} b A-4 b^{2}\left(2 A F^{\prime}+B F\right)+\frac{Q}{F}, \\
\text { 以及 } D & =A\left(F F^{\prime}\right)^{\prime}+B F F^{\prime}+C F^{2} .
\end{aligned}
$$

类似地, 由于 $(31)$ 式左端不依赖于 $x$ 和 $y$, 故有

$$
\begin{aligned}
& E=0, \quad G^{\prime} F+2 a G=0, \quad G^{\prime} f+2 b H=0, \\
& H^{\prime} F+2 a H=0, \quad 2 b G+I^{\prime} F=0,
\end{aligned}
$$

即

$$
\begin{aligned}
& E=0, \quad G=c_{0} e^{-2 a \int^{u} \frac{d z}{F(z)}}, \\
& H=c_{1} e^{-2 a \int^{u} \frac{d z}{F(z)}}, \quad I=\frac{a}{b} c_{0} e^{-2 a \int^{u} \frac{d z}{F(z)}}+c_{2},
\end{aligned}
$$

其中 $c_{0}=b c_{1} / a$.

当 $a b>0$ 时, 从 (30) 式中解得

$$
\begin{aligned}
& f(x)=-\frac{1}{a} \ln \left|\cos \lambda a\left(x+x_{0}\right)\right|, \\
& g(y)=-\frac{1}{a} \ln \left|\cos \lambda a\left(y+y_{0}\right)\right|,
\end{aligned}
$$

其中 $\lambda^{2}=b / a$. 
当 $a b<0$ 时有

$$
\begin{aligned}
& f(x)=-\frac{1}{a} \ln \left|\sinh \lambda a\left(x+x_{0}\right)\right|, \\
& g(y)=-\frac{1}{a} \ln \left|\sinh \lambda a\left(y+y_{0}\right)\right|,
\end{aligned}
$$

其中 $\lambda^{2}=-b / a$. 将 (34) 式或 (35) 式代入 (31) 式中, 得

化简 (32) 式, 得到

$$
h^{\prime}(t)=\frac{b^{2}}{a^{2}} c_{1} e^{-2 a h(t)}+c_{2} .
$$

$$
\begin{aligned}
& E(u)=-3 a(2 a J+a K+D)-[F(2 a J+a K+D)]^{\prime}, \\
& G(u)=-4 b(2 a J+a K+D)-2 a b K-2 b(F J+F K)^{\prime}, \\
& H(u)=-2 a(a K+D)-2 a(a F K+F D),
\end{aligned}
$$

其中 $J=J(u)=a A+A F^{\prime}, K=K(u)=A F^{\prime}+B F$.

将 (36) 式代入 (33) 式, 得

$$
\begin{aligned}
& J(u)=a A+A F^{\prime}=\frac{1}{2 a F} e^{-a \int^{u} \frac{d z}{F(z)}}\left[-c_{2} e^{-2 a \int^{u} \frac{d z}{F(z)}}-\frac{c_{1}}{2} \int^{u}\left(e^{-a \int^{v} \frac{d z}{F(z)}}\right) d v+c_{3}\right], \\
& K(u)=A F^{\prime}+B F=\frac{1}{F} e^{-2 a \int^{u} \frac{d z}{F(z)}}\left\{-\int^{u}\left[\frac{2 c_{2}}{F} e^{-a \int^{v} \frac{d z}{F(z)}}+e^{2 a \int^{v} \frac{d z}{F(z)}}(F J)^{\prime}\right] d v-\frac{c_{0}}{2 b} u+c_{4}\right\}, \\
& D(u)=A\left(F F^{\prime}\right)^{\prime}+B F F^{\prime}+C F^{2}=\frac{c_{2}}{F} e^{-3 a \int^{u} \frac{d z}{F(z)}}-2 a J(u)-a K(u) .
\end{aligned}
$$

因此我们得到 4 个关于 $A, B, C, Q, F$ 的方程, 而 $A, B, C, Q, F$ 均是待定的. 由此我们可 以构造方程 (1) 的一系列不变集和精确解.

综上所述, 我们将伸缩群的概念和方法进行了推广, 并用于构造 $1+2$ 维一般薄膜方程的 精确解. 在参考文献 $[6,7]$ 中, 作者给出了某些 $1+2$ 维发展方程的许多有意义的精确解, 其中 包括多项式形式的解. 因此一个自然的想法是是否这些解也可以通过本文的方法或者条件对 称的方法给出. 另外, 本文的方法也有可能通过叶状结构群方法推广得到, 关于叶状结构群方 法可以参见文献 [27-29].

\section{参 考 文 献}

1 Galaktionov V A. Groups of scaling and invariant sets for higher-order nonlinear evolution equations. Diff Integer Equation, 2001, 14(8): 913-924

2 Galaktionov V A. Ordered invariants sets for nonlinear evolution equations of KDV-type. Compute Math Phys, 1999, 39(9): 1564-1570

3 Qu C Z, Estevez P G. Extended rotation and reduction of evolution equations. Nonlinear Anal TMA, 2003, 52(6): 1655-1673

4 Qu C Z. Symmetries and solutions to the thin film equations. J Math Anal Appl, 2006, 317(2): 381-397

5 King J R. Two generalizations of the thin film equation. Math Comp Mod, 2001, 34(7-8): 737-756

6 Betelü S I, King J R. Explicit solutions of a two-dimensional fourth-order nonlinear diffusion equation. Math Comp Mod, 2003, 37(3-4): 395-403

7 King J R. Exact polynomial solutions to some nonlinear diffusion equations. Physica D, 1993, 64(1-3): $35-65$

8 Bluman G W, Cole J D. The general similarity solution of the heat equation. J Math Mech, 1969, 18(1): 1025-1042 
9 Clarkson P A, Mansfield E L. Symmetry reductions and exact solutions of a class of nonlinear heat equations. Physica D, 1993, 70(3): 250-288

10 Estevez P G. The direct method and the singular manifold method for the Fitzhugh-Nagumo equation. Phys Lett A, 1992, 171(5-6): 259-261

11 Fuschych W I, Zhdanov R Z. Anti-reduction and exact solutions of nonlinear heat equations. J Nonlinear Math Phys, 1994, 1(1): 60-64

12 Fokas A S, Liu Q M . Nonlinear interaction of traveling waves of nonintegrable equations. Phys Rev Lett, 1994, 72(21): 3293-3296

13 Zhdanov R Z. Conditional Lie-Bäcklund symmetry and reduction of evolution equation. J Phys A Math Gen, 1995, 28(13): 3841-3850

14 Cherniha R, Serov M. Symmetries, ansätze and exact solutions of nonlinear second-order evolution equations with convection terms. Euro J Appl Math, 1998, 9(5): 527-542

15 Cherniha R, Henkel M. On nonlinear partial differential equations with an infinite-dimensional conditional symmetry. J Math Anal Appl, 2004, 298(2): 487-500

$16 \mathrm{Qu} \mathrm{C}$ Z. Group classification and generalized conditional symmetry reduction of the nonlinear diffusionconvection equation with a nonlinear source. Stud Appl Math, 1997, 99(2): 107-136

$17 \mathrm{Qu} \mathrm{C} \mathrm{Z}$. Exact solution to nonlinear diffusion equations obtained by a generalized conditional symmetry method. IMA J Appl Math, 1999, 62(3): 283-302

$18 \mathrm{Qu} \mathrm{C} \mathrm{Z}$. Classification and reduction of some systems of quasilinear partial differential equations. Nonlinear Anal TMA, 2000, 42(2): 301-327

19 Qu C Z, Zhang S L , Liu R C. Separation of variables and exact solutions to quasilinear diffusion equations with nonlinear source. Physica D, 2000, 144(1-2): 97-123

20 Zhang S L, Lou S Y, Qu C Z. New variable separation approach: Application to nonlinear diffusion equations. J Phys A Math Gen, 2003, 36(49): 12223-12242

$21 \mathrm{Qu} \mathrm{C} \mathrm{Z}$. Reductions and exact solutions of some nonlinear partial differential equations under four types of generalized conditional symmetries. J Aust Math Soc B, 1999, 41(1): 1-40

$22 \mathrm{Qu} \mathrm{C} \mathrm{Z,} \mathrm{Estevez} \mathrm{P} \mathrm{G.} \mathrm{On} \mathrm{nonlinear} \mathrm{diffusion} \mathrm{equations} \mathrm{with} x$-dependent convection and absorption. Nonlinear Anal TMA, 2004, 57(4): 549-577

23 Zhang S L, Lou S L, Qu C Z. On nonlinear diffusion equations with x-dipendent covetion and absorption. J Phys A Math Gen, 2003, 36: 12221-12242

24 Zhang S L, Lou S L, Qu C Z. Variable Separation and exact solutions to generalized nonlinear diffusion equations. Chin Phys Lett, 2002, 19(12): 1741-1744

25 Goard J M. The method of generalized conditional symmetries and its various implementations. Euro J Appl Math, 2000, 11(2): 215-224

26 Basarab-Horwath P, Zhdanov R Z. Initial-value problems for evolution partial differential equations and higher-order conditional symmetries. J Math Phys, 2001, 42(2): 376-389

27 Zhdanov R Z. Higher conditional symmetry and reduction of initial value problems. Nonlin Dynamics, 2002, 28(1): 17-27 (2002).

28 Vessiot E. Sur I'integeration des sistem differential qui admittent des groupes continus de transformations. Acta Math, 1904, 28(2): 307-319

29 Martina L, Sheftel M B, Winternitz P. Group foliation and non-invariant solutions of the heavenly equation. J Phys A Math Gen, 2001, 34(43): 9243-9263

30 Anco S C, Liu S. Exact solutions of semilinear radial wave equations in $n$ dimensions. J Math Anal Appl, 2004, 297(1): 317-342 